\title{
UNA APROXIMACIÓN CRÍTICA A LA TEORÍA DE LA ACELERACIÓN DE HARTMUT ROSA
}

\author{
A Critical Approach to Hartmut Ros a's AcCeleration Theory \\ Paula Duerto Porquet
}

DOI: 10.26754 /ojs_arif/a.rif.202014534

\begin{abstract}
RESUMEN
Este trabajo analiza críticamente las ideas fundamentales de la teoría de la aceleración social expuesta por Hartmut Rosa en Alienación y aceleración (2016). Se destaca la ausencia de un análisis del capitalismo como motor de la aceleración. Esta ausencia lastra también las alternativas a la alienación que Rosa propone en Resonancia (2019).
\end{abstract}

PALABRAS CLAVE: Hartmut Rosa, alienación, aceleración, resonancia.

\section{ABSTRACT}

This paper critically analyzes the main ideas of the theory of social acceleration exposed by Hartmut Rosa in Alienation and acceleration. The absence of an analysis of capitalism as a cause of acceleration is criticized. This absence also negatively affects the alternatives to alienation that Rosa proposes in Resonance.

KEYWORDS: Hartmut Rosa, alienation, acceleration, resonance.

\section{INTRODUCCIÓN}

Alienación y aceleración (2016) es un ensayo breve que ofrece un análisis sociológico de las características de la temporalidad moderna. Su autor, Hartmut Rosa, es un filósofo y sociólogo alemán, catedrático de Sociología y director del instituto Max Weber, considerado como uno de los mayores exponentes contemporáneos de la Teoría Crítica.

En Alienación y aceleración (2016), Rosa aborda con herramientas sociológicas lo que muchos otros autores han apuntado de forma más intuitiva: la aceleración 
creciente de nuestra sociedad. Las páginas de la obra son ágiles y claras, y hacen accesible la reflexión sobre la temporalidad contemporánea a un público amplio. Además, aportan nuevas categorías, conceptos y marcos para el estudio de la temporalidad moderna.

El objetivo del ensayo, en palabras del autor, es reconectar la filosofía social y la sociología con las experiencias de la gente en las sociedades tardomodernas. Rosa realiza asimismo una actualización de la Teoría Crítica en torno a los conceptos de aceleración, alienación y vida buena.

Las contribuciones teóricas de Rosa son múltiples y valiosas, pero en esta nota crítica me centraré en dialogar con los aspectos de su teoría que considero menos consistentes. Mi análisis se centrará fundamentalmente en los contenidos de Alienación y aceleración (2016), aunque en el último apartado me referiré también a su obra Resonancia (2019).

\section{LA ACELERACIÓN Y SUS CAUSAS}

Alienación y aceleración se hace eco de la frase de Thomas H. Eriksen que sentencia que "la modernidad es velocidad" (2001: 15 cit. en Rosa, 2016:17). Actualmente vivimos en la era de la comida rápida, las citas express, la comunicación instantánea, la hipermovilidad, la inestabilidad laboral y el estrés. Esta atmósfera ha sido abordada por múltiples filósofos y sociólogos en las últimas décadas, desde distintas perspectivas. Algunos ejemplos son los trabajos de Bauman sobre la modernidad líquida, el estudio de Jonathan Crary de la temporalidad 24/7 (2015) o los textos de Byung-Chul Han sobre la sociedad del cansancio.

En el trabajo de Rosa, la noción de "aceleración" aspira a condensar la lógica de la modernidad tardía, aunque abarca un conjunto de fenómenos diversos. Para alcanzar una mayor precisión conceptual, el filósofo opta por dividir el concepto en tres categorías: aceleración tecnológica, aceleración del cambio social y aceleración del ritmo de vida.

En primer lugar, la aceleración tecnológica remite al "aumento deliberado de velocidad de los procesos orientados a metas específicas del transporte, la comunicación y la producción" (Rosa 2016: 21). La aceleración social, por su parte, describe la contracción del presente ${ }^{1}$ : el fenómeno según el cual las expectativas sobre el futuro cada vez son más falibles y abarcan un lapso más breve de tiempo (Id. 25). En otras palabras, nombra cómo las grandes transformaciones sociales se

1 Rosa cita a Hermann Lübbe como autor original del concepto (2009, cit. en Rosa 2016: 25). 
suceden cada vez más deprisa, sin que resten apenas periodos de estabilidad entre una y otra. Prueba de ello es que las instituciones que organizan la producción y la reproducción (la familia y el empleo) ya no cambian a una velocidad intergeneracional o generacional, sino intrageneracional (Id. 27). En tercer y último lugar, la aceleración del ritmo de vida consiste en la asunción subjetiva de esta velocidad social. La interiorización de este ritmo se manifiesta como hambre de tiempo o timefamine: el deseo o necesidad, propio de los individuos modernos, de "hacer más cosas en menos tiempo" (Rosa 2016: 31).

Tras establecer este esquema general de la aceleración, Rosa investiga sus causas y determina que los motores que originan la aceleración en la modernidad tardía son dos: uno se ubica en la esfera social y otro, en la cultural.

El motor social, por un lado, se apoya en la lógica de competición entre individuos, que permea todas las esferas sociales, empujando a los sujetos a ser los más rápidos para no quedarse atrás. El motor cultural, por otro lado, se basa en la premisa culturalmente arraigada de que debemos aprovechar al máximo todas las oportunidades que se nos presentan, con el objetivo de alcanzar una vida plena: "en la sociedad secular moderna, la aceleración sirve como el equivalente funcional de la promesa (religiosa) de vida eterna" ${ }^{2}$. (Rosa 2016: 47).

No obstante, el filósofo subraya que, en la modernidad tardía, la aceleración ha alcanzado un punto de desarrollo cuasiautónomo. En consecuencia, ya no precisa de fuerzas externas para seguir creciendo, porque los tres tipos de aceleración descritos se retroalimentan entre sí (Rosa 2016: 50).

\subsection{Capitalismo, tecnología y aceleración}

Lo llamativo del análisis de Rosa es que, aunque la circunvala constantemente, no llega a abordar directamente la relación entre capitalismo y aceleración. Tanto el motor social como el motor cultural se nutren de lógicas capitalistas y son incomprensibles al margen de estas. Sin embargo, y si bien Rosa reconoce esta relación en algunos pasajes, no concede a la economía la importancia suficiente como para abordarla en tanto que motor aceleratorio per se.

Como resultado, el tratamiento de la relación entre crecimiento, desarrollo y aceleración social resulta confuso. Su investigación no distingue entre crecimiento económico y desarrollo tecnológico, y por consiguiente no logra esclarecer la

\footnotetext{
2 La pulsión que Rosa describe presenta muchos paralelismos con la patología social contemporánea, atribuida fundamentalmente a los adolescentes, conocida como FOMO.
} 
conexión entre ambos. En el primer capítulo, Rosa utiliza la noción de "aceleración tecnológica" en un sentido abstracto, escindido de las lógicas capitalistas que de facto promueven la innovación técnica (Rosa 2016: 34-39).

Desde esta perspectiva ideal, "la sociedad moderna (...) se caracteriza por un aumento en el ritmo de vida (o escasez de tiempo) a pesar de las tasas de aceleración tecnológica tan impresionantes" (Rosa 2016: 39). Rosa entiende que, en el plano lógico, los avances tecnológicos deberían reducir la cantidad de tiempo que necesitamos para llevar a cabo nuestras tareas cotidianas. Así, la aceleración tecnológica debería ser sinónimo de un incremento del tiempo libre y una sociedad menos acelerada. Evidentemente, esto no es así en la práctica, pero su análisis elude las razones económico-políticas de esta disonancia y se ciñe a los dos motores descritos (social y cultural) para explicarla.

Esta postura muestra claras inconsistencias. Calificar la relación entre crecimiento y aceleración de paradójica solo puede hacerse a costa de omitir por completo la naturaleza del crecimiento en un sistema capitalista. En un marco capitalista, la producción y el desarrollo técnico no se orientan a la satisfacción de necesidades humanas, sino al incremento constante de los beneficios privados. Por lo tanto, tal y como Moruno (2019) señala, "la reducción del trabajo socialmente necesario no desemboca mecánicamente en el ocio aristotélico para la multitud" (Rosa 2016: 83).

Rosa está en lo cierto cuando defiende que el avión, el teléfono o el ordenador personal no pueden explicar por sí mismos la aceleración social. No obstante, durante gran parte de su análisis obvia las lógicas económicas en las que se enmarca la producción y distribución de estos aparatos, las cuales condicionan desde el principio su uso e impacto social. En definitiva, la potencia de su análisis queda mermada por su incapacidad de reconocer el capitalismo como gran motor de la aceleración.

Junto con esta debilidad, existe otro punto ciego en su teoría: la ausencia de un análisis de los rasgos específicos del capitalismo digital. En Alienación y aceleración, Rosa defiende que no existe ninguna propiedad en los aparatos tecnológicos mismos que nos impulse a utilizarlos de forma compulsiva (Rosa 2016: 40). Quizás esto sea en gran medida cierto para aparatos como el tren, el microondas o el teléfono fijo. No obstante, algunos autores apuntan que en las últimas décadas se ha producido un cambio de paradigma en la historia de la tecnología. En el paradigma actual, el rasgo distintivo es el desarrollo de los mecanismos de control. Esto hace que resulte ingenuo considerar que "las condiciones tecnológicas contemporáneas son esencialmente un conjunto neutral de herramientas que pueden 
utilizarse de maneras diferentes" (Crary 2015: 56). Por el contrario, los aparatos tecnológicos digitales tienen una naturaleza muy específica:

En lugar de ser un medio para un conjunto más amplio de fines, el aparato es el fin en sí mismo. Su propósito es dirigir al usuario a la realización cada vez más eficiente de las tareas y funciones rutinarias de la máquina misma. (Crary 2015: 54).

La escritora Marta Peirano analiza esta misma cuestión en El enemigo conoce el sistema (2019). El primer capítulo de la obra, titulado "Adicción", detalla la aplicación de los hallazgos de la psicología conductual en las nuevas tecnologías:

Todos y cada uno de esos servicios [digitales] tiene una función y un objetivo muy concretos y ninguno es mejorar nuestra vida (...). Cada pequeño aspecto de su funcionamiento ha sido diseñado por expertos en comportamiento para generar adicción. ${ }^{3}$ (Peirano 2019: 22)

Además, la tecnología digital no solo se caracteriza por generar adicción sino por utilizar esa adicción para rentabilizar económicamente el tiempo que el usuario dedica al aparato: "su política es acumular la mayor cantidad posible de esa información para vendérsela al mejor postor. Somos el producto” (Peirano 2019: 22).

En conclusión, actualmente se hace más difícil que nunca sostener que la tecnología sea política o económicamente neutral, como argumenta Rosa. Por el contrario, juega por sus propias características un papel importante en la configuración de la temporalidad contemporánea, que en la obra de Rosa queda inexplorado.

\section{UNA CRÍTICA A LA ACELERACIÓN BASADA EN EL CONCEPTO DE ALIENACIÓN}

En Alienación y aceleración, Rosa elabora una crítica de la aceleración que presenta tres variantes: funcionalista, moral y ética. De todas ellas, su ensayo se centra fundamentalmente en la tercera. Rosa lleva a cabo una actualización de la Teoría Crítica centrada en el concepto de alienación, y apuesta por fundamentar la crítica social en una base inmanente, conectada con las experiencias de las personas. Para

\footnotetext{
3 Tristan Harris, antiguo trabajador de Google, coincide con ella en el diagnóstico: "Puedes decir que es mi responsabilidad ejercitar un cierto autocontrol sobre el uso de mis dispositivos digitales, pero no estarías reconociendo que hay un millar de personas al otro lado de la pantalla cuyo trabajo es acabar con cualquier asomo de responsabilidad que me quede" (Bosker 2016 cit. en Peirano 2019: 52).
} 
ello, se apoya tanto en el malestar social existente como en la noción de vida buena que guía implícitamente a los sujetos modernos. Sostiene que ambos elementos se revelan en "los sentimientos (contradictorios), convicciones y acciones de los propios actores sociales" (Rosa 2016: 85).

Partiendo de esa base, Rosa hace notar, en la primera parte de su crítica, la contradicción entre la aparente sensación de libertad presente en la modernidad y la retórica del deber que sutilmente configura nuestra cotidianeidad. El imperio de los horarios, los plazos y el poder de lo inmediato tiene "el efecto abrumador de crear sujetos de culpa", que nunca pueden cumplir las expectativas propias o ajenas, y que se encuentran desbordados por listas infinitas de tareas por hacer (Rosa 2016: 131). La culpa que provoca esta situación, a diferencia de la católica, no ofrece acceso al perdón, pues el fracaso se entiende como una responsabilidad netamente individual. Por lo tanto, la única forma de remediarlo es seguir tratando de incrementar nuestra eficiencia. En última instancia, la aceleración nos ha llevado a "la heteronomía total, a la inversión radical de la promesa de la modernidad" (Id. 143).

La segunda parte de la crítica ética, y la más importante, se apoya en el concepto de alienación, contrapuesto al de vida buena. La alienación se clasifica en cinco formas de relación: alienación del espacio, de las cosas, de las acciones, del tiempo, y del yo y los otros. (Rosa 2016: 146-174). A continuación, explicaré brevemente en qué consiste cada una de ellas.

La primera forma de alienación, respecto del espacio, tiene que ver con los efectos de la hipermovilidad y el desarraigo, y con la consiguiente pérdida de familiaridad y significado del espacio. La alienación respecto de las cosas, por otra parte, es el resultado del consumismo acelerado, la obsolescencia prematura y la cultura de usar y tirar. Impide establecer relaciones significativas con las cosas, puesto que estas solo poseen valor en función de su novedad. En tercer lugar, la alienación de las acciones designa la dificultad o imposibilidad de encontrar tiempo para hacer lo que realmente se quiere hacer, o de tomar decisiones conscientes e informadas. En el mundo de la velocidad, buscamos la satisfacción de los deseos a corto plazo (como navegar por internet), aunque los placeres que ofrecen una satisfacción postergada (como tocar el violín) nos resulten en el fondo más placenteros ${ }^{4}$. Tenemos miedo a perder oportunidades constantemente y eso nos empuja a intentar abarcar más cosas de las que realmente podemos digerir. Como

4 Peirano (2019) explica este fenómeno en términos de una adicción generalizada a la dopamina, promovida por la industria alimenticia, tecnológica y cultural. 
resultado, tenemos una concepción de lo que somos o lo que nos gusta que no se corresponde en absoluto con nuestros hábitos cotidianos (Rosa 2016: 163).

La penúltima forma de alienación tiene que ver con el tiempo. Consiste en el aplanamiento o vaciado de las experiencias, en buena medida como consecuencia de la invasión de las pantallas y los dispositivos en nuestra cotidianeidad. Habitualmente, la relación entre el tiempo de la experiencia y del recuerdo es inversa: un momento bueno pasa rápido, pero deja un recuerdo dilatado; por el contrario, un momento aburrido se vive lentamente, pero deja un recuerdo breve. Sin embargo, las actividades que ocupan gran parte de nuestra vida diaria, como navegar por internet, operan con una relación distinta. El patrón temporal es corto-corto: el tiempo pasa rápidamente durante la actividad, y después tampoco deja huella. Es como si esos momentos se hubieran evaporado, provocando una desagradable sensación de vacuidad en el sujeto. Según Rosa, este es el patrón temporal característico en la modernidad tardía. Por eso, muchos teóricos hablan de un tiempo que "se va quedando sin memoria" (Id. 169). O, en palabras de Walter Benjamin, de una época de las Erlebnisse (vivencias) frente a las Erfahrungen (experiencias significativas y transformadoras) (Ibid.).

Por último, la forma de alienación vinculada al yo y los otros se corresponde con la erosión del compromiso, la saturación social y la falta de profundidad en nuestras relaciones. Las cinco formas de alienación descritas por Rosa integran un concepto amplio que puede sintetizarse como un "silenciamiento del mundo" o una "relación de la ausencia de relación" (Rosa 2019a: 75).

La elaboración de Rosa del concepto de alienación es interesante y valiosa, tanto desde una perspectiva ética como descriptiva. No obstante, como expondré en el próximo apartado, presenta carencias significativas si pretende establecerse como el vector nuclear de la crítica social. A continuación, presentaré el equivalente positivo de la alienación, la resonancia, y expondré las insuficiencias teóricas que ambas comparten, en tanto que criterios éticos con una pretensión omniabarcante.

\section{AliENACIÓN Y RESONANCIA}

\subsection{Límites éticos de la teoría de la resonancia}

En Alienación y aceleración, Rosa contrapone el concepto de alienación al de resonancia, aunque apenas llega a esbozar el segundo en esta obra. La noción de resonancia se encuentra desarrollada en profundidad en una obra posterior, a la que da título. Esta noción constituye el núcleo de lo que el autor entiende por una vida buena, y se define a grandes rasgos de la siguiente forma: 
La resonancia implica, en primera línea y, ante todo, un estado o modo de relación dinámica con el mundo en la cual este (en tanto algo en cada caso particular que nos encuentra como mundo) y el sujeto se conmueven y transforman recíprocamente. (Rosa 2018: 75)

Algunos ejemplos de esta forma de relación serían "un intercambio de miradas o un diálogo en el que ambos hablantes se escuchan y responden recíprocamente", así como el efecto que se produce a veces "cuando se aprende a tocar un instrumento, cuando saltamos al océano y nadamos en él o cuando horneamos pan” (Rosa 2019a: 75).

El concepto resulta interesante si se entiende como una herramienta filosófica que describe experiencias opuestas a la sensación cotidiana de letargo. Los textos de Rosa sobre la resonancia son sugerentes y proporcionan pistas para repensar nuestras vidas desde una nueva perspectiva. Asimismo, ofrecen una terminología analítica que permite nombrar sensaciones o fenómenos que se encuentran habitualmente fuera del debate ético y político.

Ahora bien, si la resonancia pretende arrogarse el título de remedio total contra la alienación, o, en términos de Rosa, de "metacriterio" para la crítica social, para la definición de una vida buena y para la proyección de nuevos horizontes políticos, entonces se revela insuficiente. La noción de resonancia de Rosa, al igual que la de alienación, relega las necesidades básicas y los aspectos más materiales de la existencia a un segundo plano; deja al margen la crisis ecológica a la que nos enfrentamos y su necesaria vinculación con la ética; y, finalmente no ofrece fundamentos sólidos para criticar la desigualdad social. Estas carencias no serían problemáticas si las pretensiones del concepto fueran parciales, pero Rosa aspira a desarrollar una crítica social "total", que sin embargo no logra abarcar algunas cuestiones de vital importancia en la actualidad.

5 En el epílogo de Resonancia, Rosa defiende la resonancia desde el "monismo normativo". Entiende que esta noción "no precisa ser complementada con una crítica de las condiciones de reconocimiento, de distribución, de entendimiento o de productividad, porque las incluye" (Rosa, 2019b: 576). Según Rosa, la noción de Resonancia puede abarcar la crítica a cualquier experiencia. Por ejemplo, una persona sin hogar, que tiene hambre y frío, podría criticar su situación en términos de falta de resonancia, puesto que desde su posición el mundo se presenta como algo hostil, lo cual constituye una forma de relación con el mundo carece de resonancia. No obstante, esta justificación resulta forzada y poco convincente. 


\subsection{Límites políticos de la teoría de la resonancia}

El segundo problema que plantea la resonancia como propuesta frente a la aceleración y la alienación es que nace de un enfoque que contradice su análisis y su crítica. En Alienación y aceleración, Rosa estudia de qué forma las estructuras sociales son las responsables de generar una discordancia entre las aspiraciones de los sujetos y las condiciones de posibilidad que los rodean. Describe, asimismo, cómo la aceleración de la sociedad conduce a la alienación generalizada. Además, critica la producción de "sujetos de culpa" mediante la creencia impuesta de que la incapacidad de ajustarse a las normas temporales reside en el individuo y no en las exigencias sociales inalcanzables. Finalmente, denuncia que el régimen temporal opera como un poder totalitario, situado al margen de cualquier control político democrático. Partiendo de esta crítica a la aceleración social, cabría deducir que la propuesta que se seguiría de la misma sería la transformación de las estructuras y normas sociales que la originan. Esto tendría el objetivo de desacelerar la sociedad y favorecer las condiciones para desarrollar una vida buena.

Sin embargo, su teoría de la alienación y la resonancia privilegia un enfoque individual, que estudia las condiciones personales de la experiencia resonante, pero no reflexiona en profundidad sobre las condiciones sociales necesarias para favorecerla (Rosa 2019). Si la alienación persistente es una forma de estar en el mundo indeseable, y si su origen reside en instituciones y lógicas sociales aceleratorias, ¿no cabe deducir que es imprescindible y prioritario cambiarlas? ¿hasta qué punto es posible resistir individualmente a un régimen totalitario? ¿no precisamos, fundamentalmente, de estrategias colectivas de desaceleración?

La omisión de una reflexión política en su obra hace que su teoría corra el riesgo de ser interpretada en un sentido individualista, aunque esta no sea la intención del autor. Resulta fácil terminar la lectura de su obra con la sensación de que la solución quizás sea utilizar menos el móvil y hornear pan con más frecuencia. En este sentido, cabría incluso decir que la obra de Rosa puede llegar a generar unos nuevos sujetos de culpa que, tras tomar conciencia de la aceleración, tratan de deshacerse de ella individualmente, pero se sienten frustrados porque sus condiciones no se lo permiten.

Esta ausencia de una perspectiva política se pone de manifiesto en el primer capítulo de Alienación y aceleración. En él, Rosa sostiene que la oposición política a la aceleración social se reduce a grupos fundamentalistas con escasa relevancia social (Rosa 2016: 62). En otras palabras, no ofrece muchas esperanza sobre la posibilidad de articular una resistencia colectiva amplia. 
En contraste con este planteamiento, el sociólogo Jorge Moruno encuentra en los movimientos sociales más extendidos una oposición implícita pero activa al régimen temporal contemporáneo, así como una fuente de inspiración teórica y práctica para la crítica de la aceleración. El ecologismo sería un ejemplo claro de esta resistencia, puesto que defiende el decrecimiento y desaceleración del sistema económico. También el movimiento feminista, de acuerdo con Moruno, contiene un potencial similar:

El trabajo de cuidados reservado a las mujeres y despreciado como no productivo (salvo que se mercantilice) pone en valor todo aquello que la ley del valor desprecia. El feminismo como crítica del "ser" en la modernidad, como crítica de la economía política [propone] la posibilidad de transformación en favor de una temporalidad pensada desde las necesidades y tiempos de vida. (Moruno 2019: 59)

Aunque a priori el feminismo no se define como una lucha contra la aceleración, Moruno encuentra que sus reivindicaciones y propuestas apuntan a un horizonte vertebrado por una temporalidad muy distinta. El lema "poner la vida en el centro" alude a la necesidad de repensar la organización social desde el punto de vista de una vida que merezca la pena ser vivida, situando en el centro de la economía y de la política las tareas socialmente necesarias y deseables, en lugar de los dictados del mercado. Esta potencia se revela asimismo en muchos otros actores sociales que luchan contra la precariedad laboral, la gentrificación, el desmantelamiento de las pensiones o la privatización de los servicios públicos. Todas estas iniciativas confrontan, aunque de forma indirecta, un régimen temporal acelerado y heterónomo. En conjunto, constituyen una barrera de contención frente a la incertidumbre, el estrés y la precariedad vital que subyacen al concepto de alienación de Rosa.

En síntesis, la perspectiva de Moruno ofrece una lectura de la realidad social más optimista que la de Rosa y brinda pistas para articular estrategias colectivas contra los efectos destructivos del régimen temporal contemporáneo.

\section{Conclusiones}

Alienación y aceleración es una obra que aborda una problemática plenamente vigente con gran potencia descriptiva y analítica. Cumple el objetivo de provocar la reflexión y erigir una crítica certera contra un régimen temporal asfixiante y naturalizado. No obstante, tal como he intentado argumentar en estas páginas, algunos aspectos de su teoría resultan poco consistentes. En primer lugar, Rosa pierde potencial explicativo al evitar abordar de forma directa la evidente relación 
entre la aceleración y el sistema capitalista. En segundo lugar, su obra no investiga en profundidad el papel específico de la tecnología digital en la configuración de la temporalidad contemporánea. En tercer lugar, la noción de alienación y su contrapartida positiva, la resonancia, fracasan en la aspiración de convertirse en criterios éticos suficientes y absolutos. Por último, la obra de Rosa proporciona muchas herramientas útiles para la crítica social, pero no ofrece apenas instrumentos para traducirlas en acción política, lo cual resulta problemático para una investigación con una vocación eminentemente práctica.

Paula Duerto Porquet

Universidad de Zaragoza paulaneise@gmail.com

\section{BiBLIOGRAFÍA}

Bosker, B., "The Binge Breaker", The Atlantic (noviembre de 2016), https://www.the atlantic.com/magazine/archive/2016/11/the-binge-breaker/501122/. Consultado el 08/06/2020.

Crary, J. (2015): 24/7. El capitalismo al asalto del sueño, Barcelona: Planeta.

Moruno, J. (2019): No tengo tiempo. Geografias de la precariedad, Madrid: Akal.

Pérez Orozco, A. (2014): Subversión feminista de la economía, Madrid: Traficantes de sueños. Peirano, M. (2019): El enemigo conoce el sistema, Barcelona: Debate.

RosA, H. (2016): Alienación y aceleración. Hacia una teoría crítica de la temporalidad en la modernidad tardía, Madrid: Katz.

Rosa, H. (2019a): "La "resonancia" como concepto fundamental de una sociología de la relación con el mundo", Revista Diferencias, n. 7, pp. 73-80

Rosa, H. (2019b): Resonancia. Una sociología de la relación con el mundo, Madrid: Katz.

Rosa, H. (2019c): Remedio a la aceleración. Ensayos sobre la resonancia, Barcelona: Ned.

Rosa, H. y Bialakowsky, A. (2018): "Alienación, aceleración, resonancia y buena vida. Entrevista a Hartmut Rosa", Revista Colombiana de Sociología, vol. 41, n. 2, pp. 249-259. 